\title{
TRANSFORMATION BASED ALGORITHMS FOR CHANGE DETECTION IN FULL POLARIMETRIC REMOTE SENSING IMAGES
}

\author{
Seyd Teymoor Seydi ${ }^{1}$, Reza Shahhoseini ${ }^{1 *}$ \\ ${ }^{1}$ School of surveying and Geospatial Engineering, University of Tehran, Tehran, Iran \\ (seydi.teymoor, rshahosseini)@ut.ac.ir
}

Commission VI, WG VI/4

KEY WORDS: Polarimetric Change Detection, UAVSAR, MAD, PCA, IR-MAD, Land Cover

\begin{abstract}
:
Thanks to the recent advances in the development of polarimetric synthetic aperture radar (SAR) sensors, this remote sensing field attracts many applications. Among the different applications of these data, change detection is one of the most important applications. PolSAR images, due to interactions between electromagnetic waves and the target, could be used to study changes in the Earth's surface. This paper is a type of transformation-based method for polarimetric change detection (CD) purpose. For this purpose, we use full polarimetry imaging radar and extracted 138 features based on decomposition. The CD methods are the principal component analysis (PCA), the Multivariate Alteration Detection (MAD), the Iteratively Reweighted Multivariate Alteration Detection (IR-MAD), the Covariance Equalization (CE), and the Cross-Covariance (CRC). Assessment of the incorporated methods performed using most common criteria as quantity and quality assessment, such as overall accuracy (OA), kappa coefficient, and as visual analysis. The results of the experiments show that $\mathrm{CC}$ has better performance compared with other
\end{abstract} algorithms.

\section{INTRODUCTION}

A world in which we live is constantly changing (Seydi and Hasanlou, 2017a). With the study can understand the origin of these changes: human activities and natural phenomena (Hussain et al., 2013). This process of change and transformation causes destroys some phenomenon, and there are some phenomena and effects (Shah-Hosseini et al., 2015). These changes are, such as, the user changes cause to grow cities, soil erosion and natural events that triggered the flooding North of the fields and the sea advance or rivers (Liu, 2015). Hence, to optimize the resources management and exploit the knowledge of this process of change seems to be imperative (Seydi and Hasanlou, 2017). Remote sensing (RS) is kind of new source of many applications in the field of Earth Sciences that studies one of the most important applications identified changes to the Earth's surface(Marinelli et al., 2019). The change detection (CD) is a process usedthe differences procedure between the two different phenomena at the time of measurement (Hasanlou and Seydi, 2018).

Nowadays, with the advancement of RS technology, it is possible to get high-quality images and high spatial resolution (Bruzzone and Bovolo, 2013). Also, coming new SAR sensors improved temporal resolution that is really important for $\mathrm{CD}$ purposes. Among the type of RS Sensors the SAR sensors, due to operating independently of weather and daylight play role in RS analysis and widely used in many applications such as CD (Marinelli et al., 2017).

Recently, with increasing available to polarimetric synthetic aperture radar (PolSAR) data using them convert to hot topic among researchers in many application such as: classification, $\mathrm{CD}$, and target detection(Dabboor et al., 2019; Nielsen et al., 2019a; Sabry and Ainsworth, 2019; Silva et al., 2019; Yamaguchi et al., 2019). This data could be obtained in four different channel as VV (Vertical transmit and Vertical receive), $\mathrm{HH}$ (Horizontal transmit and Horizontal receive), VHVertical transmit and Horizontal receive), and HV (Horizontal transmit and Vertical receive) (Najafi et al., 2019). The different scattering mechanisms caused to give more details from the objects physical nature in the type of polarization. For this end, the used decomposition matrix that produces more features.

Recently, many methods have been developed for PolSAR data. However, these methods have been applied as successfully on PolSAR data and provide acceptable results but those methods are more complex for implantation(Yamaguchi et al., 2019). On the other hand, the PolSAR data due to existence high speckle noise need to a special technique for extraction change information. Also, several CD methods covert to a big challenge for users. So, it needs to consider a type of transformation based methods for CD purposes (Hasanlou and Seydi, 2018).

The transformation-based method (TB) applied in many RS analysis. Based on many research has been done the TB methods have a high potential for extraction information. And also, those are simple for implantation.

This paper considered the performance 5 the most common TB methods for the polarimetric change detection purpose. These methods include MAD, IR-MAD, CE, and CRC.

This paper is outlined as follows: Section 2 states the details of types of TB methods. Section 3 introduces study areas and high-resolution datasets. Section 4 provides the evaluation results, and the last Section 5 includes the conclusion ofexperimentation results.

\section{METHODOLOGY}

This section considers the details type of TB methods and decomposition. The CD-based on fully-polarimetry dataset applied three main steps. The first step is to produce a feature for each dataset separately using decomposition. Next step is, apply TB methods on produced decomposition features. The last step, the threshold selection and produce a binary

\footnotetext{
* Corresponding author
} 
change map. Figure 1 presents the flowchart polarimetric CD based on TB methods.

\subsection{Polarimetric Decomposition}

The main purpose of decomposition is extraction physical features and structure of ground targets(Najafi et al., 2019).

Then target backscattering features can be describe by the scattering matrix S completely:

$$
s=\left[\begin{array}{ll}
S_{h h} & S_{h v} \\
S_{\text {vh }} & S_{\text {VV }}
\end{array}\right]
$$

The polarimetric target decompositions is a modern and robust technique for extraction more details of physical characterizes ground objects based on scattering mechanism. There are many decomposition methods that this research used the most common decomposition method (Dabboor et al., 2019; Nielsen et al., 2019b; Yamaguchi et al., 2019). These methods include Cloude, Freeman, Touzi, Yamaguchi four-component, and $\mathrm{H} / \mathrm{A} / \alpha$ decompositions.

\subsection{Change Detection Methods}

The main contribution TB methods are the use of multitemporal dataset as input and to reorganize via a transformation. This transformation is based on first order (linear) or high-order statistics (nonlinear) operator such as variance, correlation, etc. The most common TB methods are PCA, CE, CRC, MAD, and IR-MAD

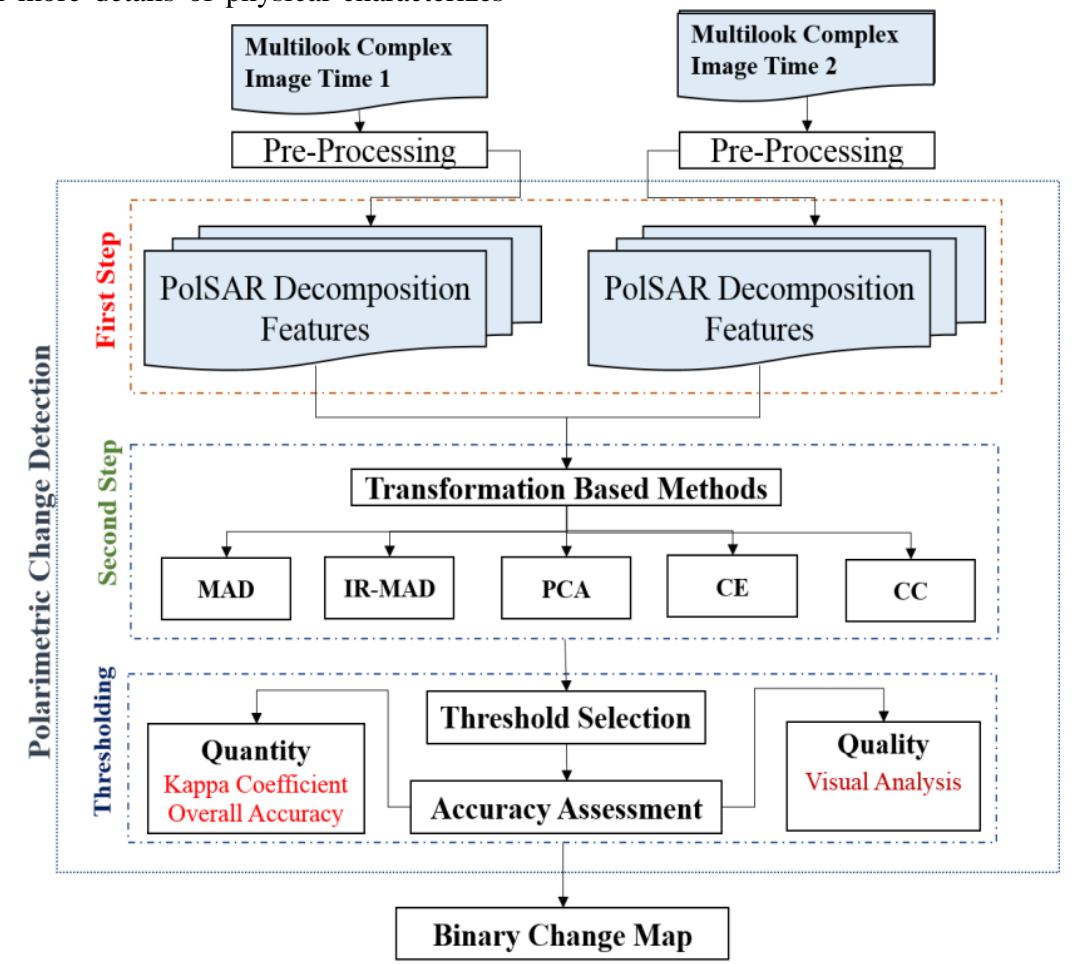

Figure 1. Flowchart Polarimetric Change Detection Based on Transformation Based Methods

2.2.1 PCA: The PCA is a linear transformation that used widely among analysis remote sensing imagery. The main purpose of PCA maintains bands have the most effects on variance (Adar et al., 2012; Eismann and Meola, 2008; Hasanlou and Seydi, 2018).
2.2.2 CRC: This method proposed by Stockham that detected changes based on second-order statics. This method tries to estimate the second time image as a linear transformation from the first time image. Finally, the change extracted by calculation residuals (Adar et al., 2012; Eismann and Meola, 2008; Pieper et al., 2015).

2.2.3 CE: The CE method is similar to the CRC method. The main different them is don't need to estimate of the cross-covariance (Eismann and Meola, 2008).

2.2.4 MAD: This method proposed by Nielsen that is based on canonical correlation analysis (CCA). The main purpose of this method is estimation a linear combination that provides high variance among corresponding bands of the multitemporal dataset (Nielsen, 2007).

2.2.5 IR-MAD: This method tries to improve the result of the $\mathrm{CD}$ based on the iterative process. The changing area becomes differ from no-change areas by giving weighting (Nielsen, 2007).

\subsection{Threshold Selection}

The threshold selection is a crucial part of the CD. Recently, a simple framework presented for threshold selection. This 
paper follows its framework for this end. In the first step, we find the minimum and maximum in the output of the TB method, and the optimum threshold is selected in the range of 0.01×(maximum-minimum) (Seydi and Hasanlou, 2017). In the next step, calculate the related accuracy based on a currently selected threshold from testing data. The optimum threshold obtained based on maximum accuracies and for the corresponding threshold.

\section{CASE STUDY}

The study area of this dataset is located in the San Francisco city. Two L-band (with a wavelength of $23.84 \mathrm{~cm}$ and a frequency of $1.26 \mathrm{GHz}$ ) fully-polarimetric images are acquired by the Jet Propulsion Laboratory/National Aeronautics and Space Administration UAVSAR. Table 1 presented characterizes of bi-temporal data. For evaluating the performance type of TB methods, it is necessary to incorporate a reference dataset as ground truth with high

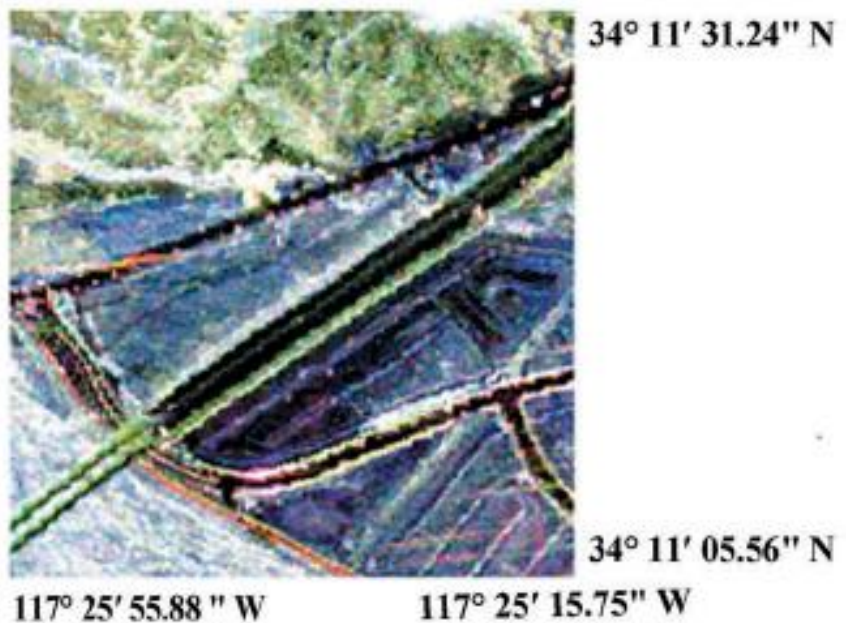

reliability. Therefore, these datasets are the availability of related ground truth data. And also, have been used in many HSCD studies (Najafi et al., 2019).

\begin{tabular}{|l|l|l|}
\hline & First Time & Second Time \\
\hline Size Data & $200 * 200$ & $200 * 200$ \\
\hline Polarization & full & full \\
\hline $\begin{array}{l}\text { Spatial Resolution } \\
\text { in Range Direction }\end{array}$ & $1.66(\mathrm{~m})$ & $1.66(\mathrm{~m})$ \\
\hline $\begin{array}{l}\text { Spatial Resolution } \\
\text { in Azimuth } \\
\text { Direction }\end{array}$ & $1(\mathrm{~m})$ & $1(\mathrm{~m})$ \\
\hline Acquired Time & $\begin{array}{l}18 \quad \text { September } \\
2009\end{array}$ & 11 May 2015 \\
\hline Band & L & L \\
\hline
\end{tabular}

Table 1. The characterizes the multitemporal polarimetric dataset

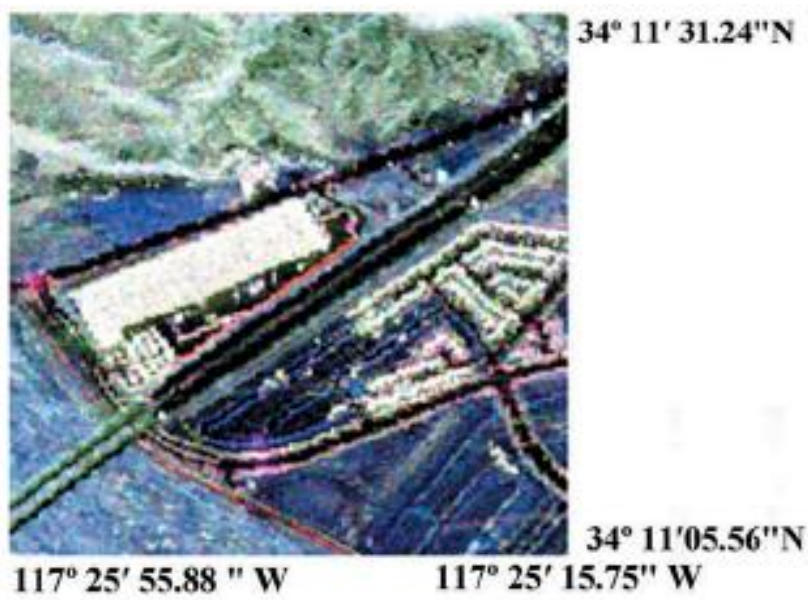

No-Change $\square$ Change

Figure 2. The (a) and (b) Pauli RGB composite of quad-pol UAVSAR images captured on 18 September 200911 May respectively, in San Francisco, United States of America, (c) ground truth, and (d) Legend

\section{EXPRIMENT AND RESULTS}

The data pre-processing plays an important role in the main process. In first, some of the most important pre-processing applied of pixel value that is de-speckles. In the next step, two data need to co-registration that applied by control points.
After of pre-processing need to extraction of polarimetric features that has been done by decomposition. The totally, 138 features extracted for each dataset.

Figure 3 presents the result of polarimetric $\mathrm{CD}$ by TB methods. Based on this figure, all of TB methods detected nearby all of the changes. This theme shows that using of TB methods have good potential for polarimetric CD purposes. 


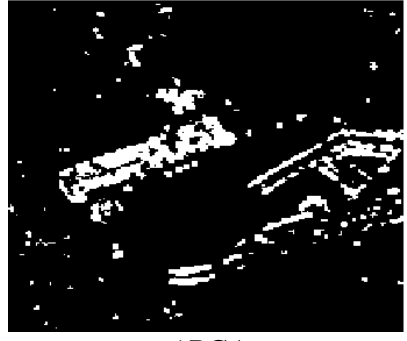

a)PCA

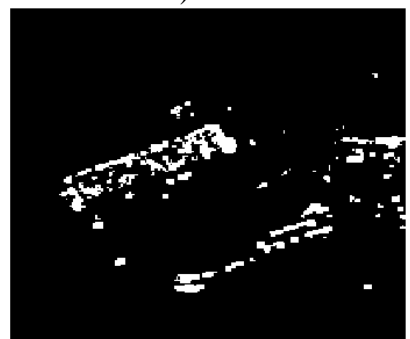

d)MAD

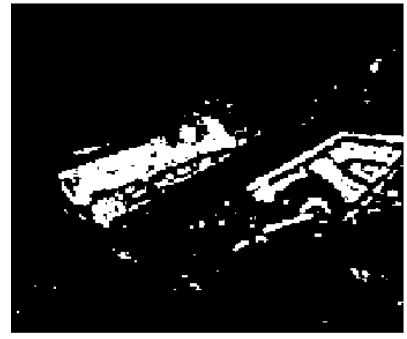

b) $\mathrm{CC}$

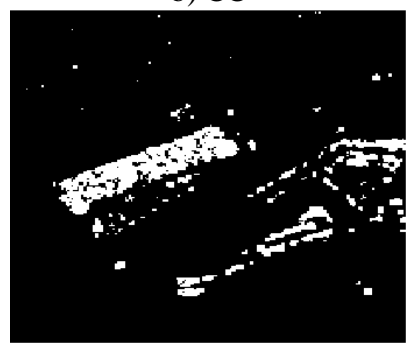

e)IR-MAD

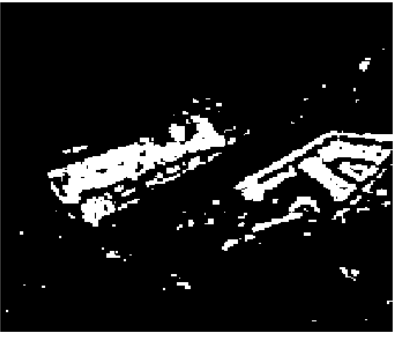

c)CE

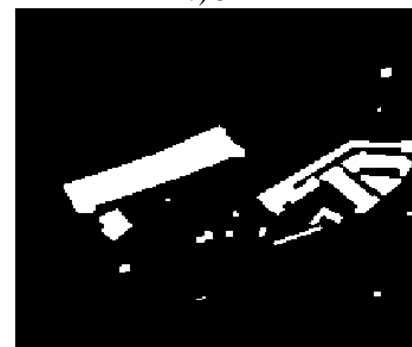

f) GT

Figure 3. The Result of Type of TB methods on a fully-polarimetric dataset.

The figure3-b and figure 3-c presented the good results for $\mathrm{CD}$ that related to $\mathrm{CC}$ and $\mathrm{CE}$ methods, respectively. The MAD algorithm has low performance compared to other methods. There are some areas that don't detect by the MAD algorithm. Also, the IR-MAD method improved results respect to a MAD algorithm that convinced performance it's on $\mathrm{CD}$.

The numerical analysis applied by the two most common indexes. Table 2 present the result of numerical analysis. Based on the presented result, all of the methods provide high accuracy as the accuracy is more than $92 \%$.

\begin{tabular}{|l|c|c|}
\hline & Overall Accuracy (\%) & Kappa Coefficient \\
\hline PCA & 93.40 & 0.5692 \\
\hline CC & 96.14 & 0.7626 \\
\hline CE & 96.01 & 0.7552 \\
\hline MAD & 92.59 & 0.3821 \\
\hline IR-MAD & 94.05 & 0.5732 \\
\hline
\end{tabular}

Table 2. The result of polarimetric CD based on TB methods.

The numerical result shows that the $\mathrm{CC}$ methods have the best performance on $\mathrm{CD}$ as provide the highest accuracy of $96.14 \%$. The similar to a visual analysis of the MAD method lowest accuracy as is lower than $93 \%$.

Both show the polarimetric SAR data has good potential for CD. Due to special content polarimetric data, the process of $\mathrm{CD}$ covert to a complex issue. So, it needs the special and robust predictor for extraction change information. For this end, the use of TB methods cans a simple solution for process data and $\mathrm{CD}$ purposes. This paper demonstrates the TB methods have high performance for $\mathrm{CD}$ purposes.

\section{CONCOLUSION}

This paper implements and evaluates TB methods between polarimetric decompositions using bi-temporal polarimetric UAVSAR images. The CD process using polarimetric SAR data applied in 3 main parts. The first step, after preprocessing the polarimetric features extracted by decomposition of the scatter matrix. The second step is to predict change area from no-change areas by TB methods. The TB methods caused the change areas to differ from nochange areas. The final step is threshold selection and optimum threshold selected and binary change map produced.

The result of the CD shows that the TB methods have high performance for polarimetric $\mathrm{CD}$. The all of methods provided the accuracy of more than $92 \%$. There is low tolerance among accuracies. The theme originated from content CD algorithms because some of the algorithms first order static properties and some methods of second order and high order static characterizes. This object caused to result of $\mathrm{CD}$ is differing. Among $5 \mathrm{~TB}$ methods, the $\mathrm{CC}$ algorithm has the highest accuracy and MAD algorithm lowest accuracy.

\section{ACKNOWLEDGMENT}

The authors would like to acknowledge the National Aeronautics and Space Administration (NASA) for providing the UAVSAR data. Also, thanks to Dr.Mehdi Hasanlu for performing pre-processing.

\section{REFERENCES}

Adar, S., Shkolnisky, Y., Dor, E.B., 2012. New approach for spectral change detection assessment using multistrip airborne hyperspectral data, in: 2012 IEEE International Geoscience and Remote Sensing Symposium. IEEE, pp. 4966-4969. https://doi.org/10.1109/IGARSS.2012.6352497

Bruzzone, L., Bovolo, F., 2013. A novel framework for the design of change-detection systems for very-highresolution remote sensing images. Proc. IEEE 101, 609-630.

https://doi.org/10.1109/JPROC.2012.2197169

Dabboor, M., Banks, S., White, L., Brisco, B., Behnamian, A., Chen, Z., Murnaghan, K., 2019. Comparison of Compact and Fully Polarimetric SAR for Multitemporal Wetland Monitoring. IEEE J. Sel. Top. Appl. Earth Obs. Remote Sens.

Eismann, M.T., Meola, J., 2008. Hyperspectral change detection: Methodology and challenges, in: IGARSS 2008-2008 IEEE International Geoscience and Remote Sensing Symposium. IEEE, $\quad$ pp. II-605. https://doi.org/10.1109/IGARSS.2008.4779065 
Hasanlou, M., Seydi, S.T., 2018. Hyperspectral change detection: An experimental comparative study. Int. J. Remote Sens. 39, 7029-7083.

Hussain, M., Chen, D., Cheng, A., Wei, H., Stanley, D., 2013. Change detection from remotely sensed images: From pixel-based to object-based approaches. ISPRS J. Photogramm. Remote Sens. 80, 91-106. https://doi.org/10.1016/j.isprsjprs.2013.03.006

Liu, S., 2015. Advanced Techniques for Automatic Change Detection in Multitemporal Hyperspectral Images. University of Trento.

Marinelli, D., Bovolo, F., Bruzzone, L., 2019. A Novel Change Detection Method for Multitemporal Hyperspectral Images Based on Binary Hyperspectral Change Vectors. IEEE Trans. Geosci. Remote Sens.

Marinelli, D., Bovolo, F., Bruzzone, L., 2017. A novel change detection method for multitemporal hyperspectral images based on a discrete representation of the change information, in: IGARSS. IEEE, pp. 161-164.

Najafi, A., Hasanlou, M., Akbari, V., 2019. Change detection using distance-based algorithms between synthetic aperture radar polarimetric decompositions. Int. J. Remote Sens. 1-14.

Nielsen, A.A., 2007. The regularized iteratively reweighted MAD method for change detection in multi-and hyperspectral data. IEEE Trans. Image Process. 16, 463-478. https://doi.org/10.1109/TIP.2006.888195

Nielsen, A.A., Skriver, H., Conradsen, K., 2019a. The Loewner Order and Direction of Detected Change in Sentinel-1 and Radarsat-2 Data.

Nielsen, A.A., Skriver, H., Conradsen, K., 2019b. Generation of Sample Complex Wishart Distributed Matrices and Change Detection in Polarimetric SAR Data, in: Accepted for International Conference on Digital Image and Signal Processing (DISP).

Pieper, M., Manolakis, D., Truslow, E., Cooley, T., Brueggeman, M., Weisner, A., Jacobson, J., 2015. Comparison of hyperspectral change detection algorithms, in: SPIE Optical Engineering+ Applications. International Society for Optics and Photonics, pp. 96110Z-96110Z. https://doi.org/10.1117/12.2188316

Sabry, R., Ainsworth, T.L., 2019. SAR Compact Polarimetry for Change Detection and Characterization. IEEE J. Sel. Top. Appl. Earth Obs. Remote Sens.

Seydi, S.T., Hasanlou, M., 2017a. A new land-cover matchbased change detection for hyperspectral imagery. Eur. J. Remote Sens. 50, 517-533.

Shah-Hosseini, R., Homayouni, S., Safari, A., 2015. A hybrid kernel-based change detection method for remotely sensed data in a similarity space. Remote Sens. 7, 12829-12858. https://doi.org/10.3390/rs71012829

Silva, C., Marino, A., Lopez-Sanchez, J.M., Cameron, I., 2019. Multi-Temporal Quad-Polarimetric Change Matrix for Agricultural Fields Monitoring.

Yamaguchi, Y., Umemura, M., Kanai, D., Miyazaki, K., Yamada, H., 2019. ALOS-2 polarimetric SAR observation of Hokkaido-Iburi-Tobu earthquake 2018. IEICE Commun. Express 8, 26-31. 